\title{
BIOCHEMISTRY OF SCHIZOPHRENIA
}

\author{
J. R. Smythies, M.D., M.R.C.P., D.P.M. \\ Department of Psychological Medicine, University of Edinburgh
}

\section{Specific Hypotheses}

THE last ten years have witnessed an enormous increase in the amount of work carried out in this field and, after many years of disappointment, at long last some positive findings are beginning to emerge. It is too early to make the claim that any definite cause of the illness is known but evidence is now to hand that suggests quite strongly the type of biochemical abnormality that may be involved. Progress in research depends to a great extent on the formulation of adequate hypotheses that can be tested experimentally. The recent progress in schizophrenia research has depended partly on the development of such hypotheses and partly on ad hoc observations both on the metabolism of schizophrenic patients and on the mode of action of psychotomimetic drugs.

\section{The First Specific Hypothesis}

One key to understanding the biochemical basis of schizophrenia may lie in the chemical formulæ of the psychotomimetic drugs. These are compounds that can induce in normal people many of the signs and symptoms of an acute schizophrenic illness without causing any delirium or clouding of consciousness. The first such drug to be discovered was mescaline in 1886 . This is a close relative of adrenaline being 3,4,5-trimethoxyphenylethylamine (Fig. I). This chemical relationship formed the basis of the first specific biochemical theory of schizophrenia published in 1952 by Osmond and Smythies. This theory postulated that schizophrenia might be caused by a derangement of adrenaline metabolism whereby the phenolic hydroxyl groups of the latter would be methylated to form dimethoxyphenylethanolamine (Fig. 2) which is a close relative of mescaline. The biochemical detail in this hypothesis was supplied by Harley-Mason. These mescaline-like products ('M-substance ') would then affect the brain and induce the psychosis. The link between stress and schizophrenia, which has been clinically demonstrated, would thus be explained, for the biochemical fault would lie in the physiological mechanisms activated by stress and thus the biochemical malfunction might only become manifest if the stress mechanisms became operative or overloaded.
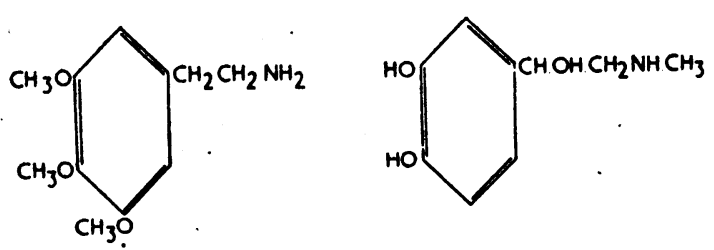

Fig. 1.-Mescaline and adrenaline.

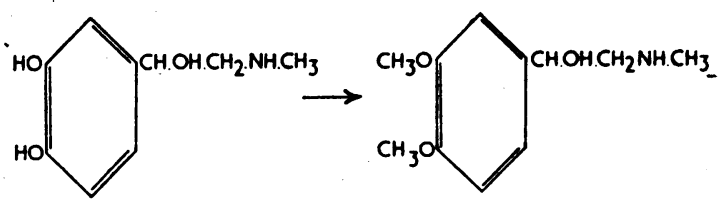

FIG. 2.-Adrenaline and dimethoxyphenylethanolamine.

Recently a fair amount of evidence has acse cumulated bearing on this hypothesis.

(i) It was demonstrated (Axelrod and Tomchick, 1958; Armstrong, McMillan and Shaw, 1957) that the normal method of metabolizing adrenaline in the human is by methylating one of the phenolic hydroxyl groups to give metanephrine (Fig. 3) which in turn is deaminated to give VMA.

(ii) It was shown by Harley-Mason, Laird and Smythies (1958) that a minor metabolite of mescaline in the human is 3 -methoxy-4,5dihydroxyphenylethylamine (Fig. 3). Thus the metabolites of mescaline and adrenaline are even more alike than their parent substances and one can see how mescaline could act by some interference between its own metabolism and that of adrenaline.

(iii) Anxiety-prone individuals, who possess a high level of tone in their sympathetic nervous systems, tend to react more severely to mescaline than do less anxiety-prone individuals and their symptoms are much more akin to those seen in actual schizophrenia. Thus a condition of stimulation of the sympathetic system seems to facilitate the psychotic process. Furthermore the symptomfree relatives of schizophrenic patients react in a much more psychotic (paranoid) manner to LSD than do normal controls.

(iv) Friedhoff and Van Winkle (1962) have claimed to have isolated dimethoxyphenylethyl- 

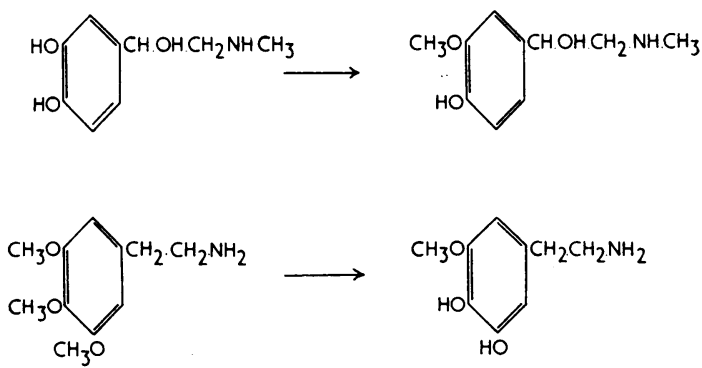

Fig. 3.-(Upper) Metabolism of adrenaline to metanephrine. (Lower) Metabolism of mescaline (a minor pathway in humans).
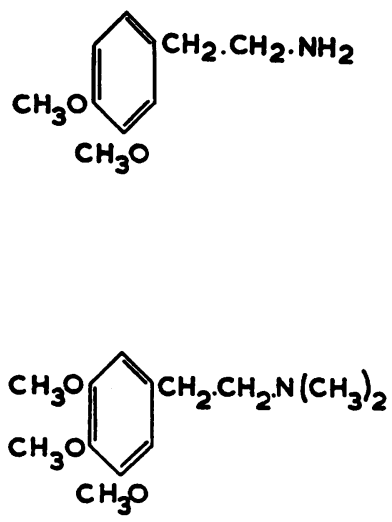

Fig. 4.-(Upper) Dimethoxyphenylethylamine. (Lower) N.N.-dimethylmescaline.

amine (Fig. 4) from the urine of 15 to 19 schizophrenics and not from any of 14 normal controls. Confirmation of this work is awaited with interest as if it is confirmed it would constitute partial verification of the Osmond-Smythies hypothesis.

(v) However, against the hypothesis is the fact that tests of overall metabolism in schizophrenia (Resnick, Wolfe, Freeman and Elmadjian, 1958; Cohen, Holland and Goldenberg, 1959; La Brosse, Mann and Kety, 196I) have failed to show any differences between the metabolism of adrenaline in the case of schizophrenic patients and normal controls. Schizophrenics excrete the same metabolites in the same proportions as do normal people; they show the same rate of utilization of injected adrenaline; nor do they apparently excrete any abnormal metabolites of adrenaline. The metabolism of dopamine, as measured by injecting its precursor DOPA, is also normal (Lenz, 1962). Nor do schizophrenics react in any qualitatively abnormal manner to injected adrenaline: they show in fact less reaction than do normal people (Pollin, Cardon and Kety, 196r; Pollin and
Goldin, 196I). However, it is known that in many cases the body deals quite differently with substances injected than with substances produced at the normal site of action of the agent and furthermore adrenaline crosses the blood-brain barrier in only a few localized places. So these negative results do not necessarily carry any great weight.

(vi) Some indirect evidence was produced by Costa (1960) who reported that there is a close quantitative correlation between the hallucinogenic powers of a drug and its capacity of potentiating the action of adrenaline on various pharmacological preparations.

(vii) The evidence concerning the general importance of excess methylation will be reviewed below.

\section{The Second Specific Hypothesis}

This was formulated independently by Gaddum and by Woolley and Shaw in 1954. This hypothesis is based on the fact that the most powerful hallucinogen known-lysergic acid diethylamide (LSD)-(Fig. 5) has powerful anti-serotonin
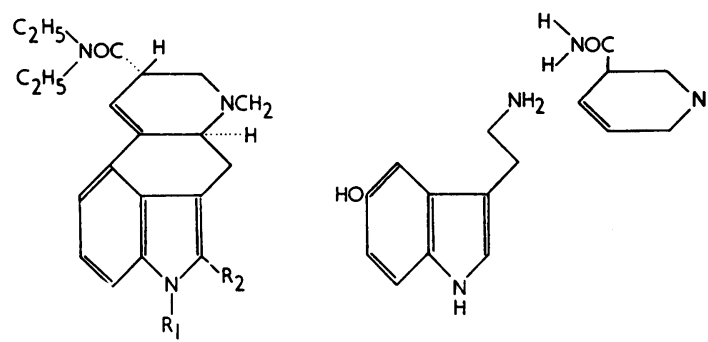

FIG. 5.-Lysergic acid diethylamine (shown as compounded of serotonin and nicotinamide).

properties when tested on smooth muscle. This led to the suggestion that psychosis may be linked with abnormalities of serotonin (5-HT) (Fig. 6, I) in the brain. We now know, however, that there cannot be any direct link between peripheral anti5-HT action and psychosis because (i) close relatives of LSD such as brom-LSD and MBL 6I are as (or more) powerful anti-5-HT agents, can cross the blood-brain barrier and yet are psychiatrically quite inert. (ii) Furthermore, no consistent major abnormalities of 5 -HT metabolism have been found in schizophrenics (Rodnight, I961). A few cases have been reported to show various quantitative abnormalities but the details require confirmation. (iii) However, 5-HT clearly must play some important role in brain function as both it and its correlated enzymes are found in the brain and in particular in those parts of the brain that are concerned with the control of emotion and 

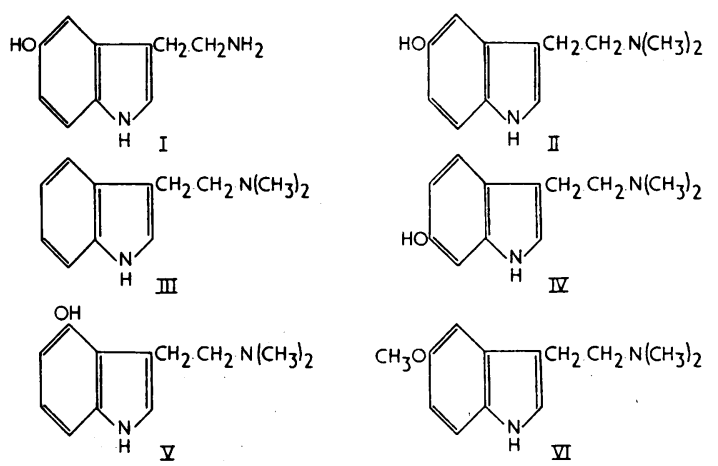

FIG. 6.-I. Serotonin. II. Bufotenin. III. DMT. IV. 6-hydroxy-DMT, a metabolite of III. V. psilocyn. VI. O-methylated derivative of bufotenin.

autonomic responses in general. Disorders of 5-HT metabolism are clearly linked with the state of the emotions. The sedative agent reserpine produces its effects on mood by depleting the brain stores of serotonin and reserpine commonly induces in humans a state of clinical depression. Furthermore, the potent anti-depressant drug imipramine facilitates the transport of $5-\mathrm{HT}$ across cell membranes and sensitizes tissues to the action of 5-HT. (iv) It has also been shown that LSD has important effects on brain 5-HT. It raises the levels of $5-\mathrm{HT}$ in the brain by $40 \%$ and induces a large increase in the rate of turnover (to $350 \%$ ) (Sankar, Gold, Phipps and Sankar, 196I). This effect is not shared by its nonpsychotomimetic relatives I-LSD and brom-LSD (Freedman, 196r). LSD and 5-HT have complex interactions in the brain. In some regions they are antagonistic, in others they are also antagonistic but reverse their roles, in yet others they act synergistically.

Brodie (1958) has put forward the hypothesis that LSD acts by depressing the central parasympathetic by competing with $5-\mathrm{HT}$ at its active sites (owing to the 'built in' indole structure of LSD) and also by activating the central sympathetic. Certainly most hallucinogens are powerful stimulants of the central sympathetic system although not all powerful central sympathetic stimulants are hallucinogenic. This attractive hypothesis still remains to be proved. But clearly $5-\mathrm{HT}$ is of great importance to psychiatry. Present trends seem to link it closer with depression than with schizophrenia but it may also play some important role in the latter.

\section{The Third Specific Hypothesis}

A third hypothesis has recently been put forward by McIsaac (196I). He notes the close chemical similarity between the hallucinogenic drug harmine

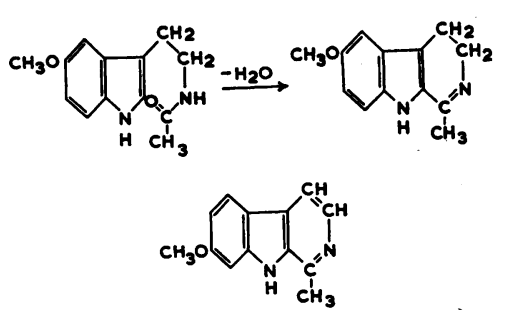

Fig. 7.-(Upper) Melatonin and ro-methoxyharmalân. (Lower) Harmine.

and the pineal hormone melatonin (Fig. $\overrightarrow{5 y}$. Melatonin is synthesized in the pineal gland ad thence is distributed to the rest of the brain. Ts function in humans is not understood. Its closge relative Io-methoxy harmalan is a potent $5-\mathrm{H}$ \% antagonist and I mg. of it completely disorganizes learned behaviour in the rat. Thus a disorder.jुf melatonin metabolism could be associated with certain cases of psychosis.

\section{The Fourth Specific Hypothesis}

The previous three hypotheses have been based on the possible production in the body of some toxic agent or on the disorder of some natumatty

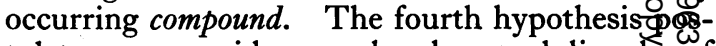
tulates a more wide-spread and general disorder of a particular biochemical mechanism that may produce a variety of abnormal substances. T尾s mechanism is methylation (Smythies, 196\$). The evidence for this is as follows: (i) We notgd earlier that mescaline can be regarded as a derivative of adrenaline when the phenolic hydro $\vec{y} 1$ groups of the latter are methylated (and one adde馬. This is O-methylation. (ii) Then a series of ot hallucinogens were discovered which are deriatives of tryptamine in which the methyl groups arye attached to the nitrogen atom (N-methylation). Tryptamine itself so treated gives N.N.-dimeth tryptamine (DMT) (Fig. 6, III), a potent halthicinogen which also causes violent disturbances of the autonomic nervous system. Its effects last for about one hour. N.N.-diethyltryptamine (DES) acts in a similar manner only the effects last about three hours. The active agents may be their 6-hydroxylated metabolites (Fig. 6, IV). Then psilocybin was discovered. This is a phosphoric acid ester of psilocyn which is 4-hydro N.N.-dimethyltryptamine (Fig. 6, V). Both these compounds are hallucinogenic with effects simifar to those produced by DET. (iv) Bufotenin (Fgy. $6, \mathrm{II})$ is N.N.-dimethyl serotonin. It has powgful effects on the autonomic system and some rather ill-defined hallucinogenic activity. \$o $\mathrm{N}$-methylation of important physiological su隹stances can produce potent hallucinogenic sub- 
stances. (v) Furthermore, if the phenolic hydroxyl group in some of these compounds is also methylated (O-methylation) (Fig. 6, VI) this increases their capacity to disrupt learned behaviour in the rat. (vi) These findings have gained much in significance by Axelrod's discovery (196r) of an $\mathrm{N}$-methylating enzyme in rabbit lung that will actually convert serotonin to bufotenin and tryptamine to DMT. Thus the metabolic mechanism has been demonstrated in mammalian tissue that will convert important bodily chemicals into psychotomimetic agents. (vii) The hypothesis that excess methylation could play some part in the ætiology of schizophrenia could be tested by feeding schizophrenics with methyl donors-that is with chemical compounds that act as donors of methyl groups in methylation processes. Methionine is just such a compound and a discovery of major importance was made by Pollin and others (I96I). They fed a variety of aminoacids to schizophrenic patients under cover of a monoamine oxidase inhibitor (to prevent the breakdown of amines derived from these amino acids). They found that methionine, and methionine alone, caused in four of nine patients an exacerbation of their symptoms. Their hallucinations, delusions and anxiety were increased and the thought disorder was exacerbated. These findings have been confirmed by Brune and Himwich (1962). Methionine has a number of properties, however, besides its action as a methyl donor. So Brune and Himwich investigated the effects of another (chemically unrelated) methyl donorbetaine. They found this to act just as methionine had done. Thus the hypothesis has survived two tests. (viii) Fischer, Lagravere, Vazquez and $\mathrm{Di}$ Stefano (196I) reported the isolation of bufotenin itself from schizophrenic urine in 25 of 26 cases. This was confirmed by Brune and Himwich (1962) but Rodnight (196r) was unable to find any in spite of a careful search using a sensitive method. In any case bufotenin could hardly be the toxic agent of schizophrenia, because people with this illness do not show the marked autonomic responses characteristic of bufotenin poisoning. But its presence in the urine, if real, may be an indication of the basic disorder of methylation processes that may constitute the metabolic basis of schizophrenia.

Thus, to summarize this section it has been shown that methylation of serotonin, tryptamine and derivatives of tryptamine and of adrenaline in either the $\mathrm{N}$ or the $\mathrm{O}$ position can produce powerful hallucinogenic substances. An enzyme capable of doing some of these reactions in vivo has been isolated. Feeding schizophrenics with methyl donors of two different chemical species (methionine and betaine; under MAOI cover) produces in many cases an exacerbation of their symptoms. Lastly the methylated compounds bufotenin and 3,4-dimethoxyphenylethylamine have been isolated, so it has been claimed, from schizophrenic urine. All these findings support the working hypothesis that a disorder of methylation processes is in some way causally connected with the schizophrenic illnesses. One difficulty here is that schizophrenia is a genetically determined condition and most if not all other genetically determined conditions are due to the lack of some enzyme, whereas here we seem to need to postulate an extra enzyme (an $\mathrm{N}$ - or Omethyl transferase) (Ashcroft, 1962). However, it is possible that some enzyme that normally keeps methylation within limits is lacking. There is one curious paradox. The general rule for these compounds seems to be that an increase in methylation is correlated with increased psychotomimetic activity. However, if we N.N.-dimethylate mescaline itself the resultant compound (Fig. 4), according to Ludueña (1935), appears to have lost its psychotomimetic activity. But, as this is based on only a single experiment, this needs confirmation.

Further experiments suggest themselves. Methionine, betaine and other methyl donors should be given together with a methyl acceptor such as glycocyamine. The hypothesis would predict that this would abolish their toxic effect on schizophrenics. Secondly, using radioactive tracer techniques a minute study should be made of methylation processes in schizophrenia. Then the presence or absence of bufotenin and dimethoxyphenylethylamine in schizophrenic urine needs settling.

\section{Empirical Discoveries \\ Carbohydrate Metabolism}

Several reports have appeared recently claiming to show that there are disorders in carbohydrate metabolism and in basic energy metabolism in schizophrenia. (i) Firstly, schizophrenics seem to show phosphorus retention. (ii) Frohman and his co-workers in a series of papers have claimed that schizophrenic plasma contains a factor with the following properties: (a) it increases the rate of turnover of ATP in red blood cells but renders this rate of turnover incapable of the large increase that is normally observed following the administration of insulin; (b) it increases the amount of glucose passing down the Meyerhof pathway of anaerobic glycolysis (which leads to the production of ATP and energy) in proportion to the amount passing down the ribose pathway (whose function is largely synthesis of nucleoproteins, etc.) but again this proportion cannot be increased by insulin as it can in normals; (c) it causes an elevation of the lactate/ 
pyruvate ratio produced by red cells suggesting some inhibition of aerobic metabolism. They localized this factor in the $\alpha$-globulin fraction of the plasma. In a recent paper they report, however, that this factor appears in the blood of schizophrenics only following physical exercise. This obscures somewhat its clinical significance. Further identification of this factor is awaited with interest. Partial confirmation of these results has been reported by Arnold and Hofmann (1962). They could not detect any differences in intermediate carbohydrate biochemistry between schizophrenics and controls in the resting state, i.e. before giving, in their case, not insulin but succinic acid. However, after giving succinic acid to the subjects (by intravenous injection) a clear difference emerged. The specific activity and $\mathrm{P}^{32}$ uptake of ATP and triose-phosphate significantly increased in the normal group but did not do so in the case of the schizophrenic group. Relatives of schizophrenics, who themselves had had no psychiatric disease, gave intermediate values. This is similar to the reaction observed by Frohman and his group following the injection of insulin. The differences before giving insulin (or succinic acid) in the two groups may possibly be explained by different exercise levels or diagnostic criteria. However, it seems probable that schizophrenics show a disturbance in intermediate carbohydrate metabolism that is apparent only after insulin or succinic acid is administered. (iii) Sacks (1959) has claimed that schizophrenic brain is unable to oxidize its proper quota of glucose and there is dilution, possibly at the pyruvate level, by intermediates derived from lipid and/or protein metabolism. (iv) This correlates with reports that a factor in schizophrenic plasma (also localized to the $\alpha$-globulin fraction) inhibits the uptake of glucose by rat diaphragm and retina.

\section{The Toxicity of Schizophrenic Body Fluids}

There have been many reports in the literature that schizophrenic body fluids are toxic to a large variety of organisms ranging from fibroblasts to monkeys. Recently more sophisticated and better controlled tests have been applied. It has been shown that schizophrenic plasma will disrupt the behaviour of trained rats to a significantly greater degree than does normal plasma. Bergen, Pennell, Freeman, Hoagland and Smythies (1960) and Winter and Flataker (1958) used the rat ropeclimbing test. The former group showed that the toxic factor will diffuse across a semi-permeable membrane. It must therefore be a small molecule attached to protein and further tests showed that the globulin fraction was mainly involved. Bishop (1960) used instrumental-conditioned behaviour with particularly good clinical control of his̊ material and showed that schizophrenic plasmo produced a significant impairment $(\mathrm{P}<0.01)$ of new learning but no impairment in the retentionof overlearning.

Using a neurophysiological method devised by Smythies, Koella and Levy (1960), Bergen: Koella, Czicmann and Hoagland (196I) showed that intravenous injections of globulin fractionso from schizophrenic plasma produce the same effects on the optic evoked potential to light flaske in the optic cortex of the unanæsthetized rabbitp (i.e. inhibition and decrease of variability) as do large doses of mescalin and LSD. German (1961 \% placed schizophrenic serum directly on the rap cortex and recorded the potential evoked in the somatosensory cortex by peripheral stimulationis. This resulted in a significantly greater potentiation of the potential as compared with the effects of normal serum. This is similar to the results produced by small doses of mescaline and $\mathrm{LSD}_{\odot}^{\circ}$ Geiger (1960) has studied the effect of schizo phrenic serum on neurones grown in tissue culture. The effects she observed were clear cuश and definite and were never seen if serum front normal people was used. (a) The glial motility (which 5-HT also effects) was increased; (b) the neural cytoplasm normally shows pumping pulsatile movements. These were increased; 就) fine undulating membranes could be observed cell surfaces and the transfer of material from oligodendroglia to neurones became more evident (d) after some time the granules of liponucleo? protein became smaller and more diffuse and migh? disappear (LSD also does this). Wada an $\vec{\Phi}$ Gibson (1959) injected extracts from schizophrenic and normal urine into the cerebrospinal fluid of monkeys and cats. The extracts from schizo phrenic urine produced a variety of abnormas behaviour patterns-e.g. states of rage and recurrent stuporose and catatonic states that were no $\underline{B}$. seen if extracts from normal urine were used. However, there is as yet no definite evidence thas these 'toxic agents' are specific for schizophrenia and indeed in some cases there is evidence that they may occur in any state of severe stress.

\section{Tryptophane Metabolism}

Price, Brown and Peters (1959) reported that 6 of I9 schizophrenics metabolized l-tryptophane्్ abnormally. They excreted excessive amounts of kynurenine and its relatives but less $\mathrm{N}$-methyl-2 pyridone-carboxamide (NMPC) than did normas controls. Similar effects were found in cases of porphyria and in other psychoses but the metabolism of tryptophane was normal in purely. neurological disorders. Benassi, Benassi, Allegr and Ballarin (r96r) were able to confirm thesळ 
results but Brown, White and Kennedy (1960) could confirm only the lesser excretion of NMPC. The significance of these findings should be clarified by further research.

\section{Tryptamine Metabolism}

Brune and Himwich (1962) have reported that schizophrenics excrete in the urine more tryptamine and indole-3-acetic acid when they are ill than they do during periods of clinical remission. Other workers have found smiliar results for catechol amines and $5-\mathrm{HT}$.

\section{Neuraminic Acid}

Bogoch, Dussik and Lever (1959) have examined a large number of schizophrenic patients and have found that they have abnormally low levels of neuraminic acid (sialic acid) in the cerebrospinal fluid. Christoni and Zappoli (1960) have confirmed this result. Bogoch and his coworkers also measured levels of hexosamine and found this to be low in schizophrenia as well as in manic-depressive psychosis and organic brain damage. Methods for measuring neuraminic acid are not yet well established, however, and this work requires extension.

\section{Histamine}

It has now also been established that schizophrenics react to histamine less than normal people do. Lucy (1954) showed this clinically in the response of the whole patient and various workers have shown this since by measuring the wheals produced by intradermal injection of histamine. This correlates with the fact that the skin of schizophrenics contains fewer mast cells than normal.

\section{Immunity and Allied Reactions}

A recent discovery of the greatest interest has been the schizophrenic and normal sera have different immunological properties (Haddad and Rabe, 1961). They sensitized guinea-pigs to schizophrenic serum and then completely desensitized them by repeated injections of normal serum. If schizophrenic serum was then injected the guinea-pigs showed anaphylactic reactions in every case. Thus schizophrenic serum has one or more antigens not present in normal serum. Fessel (I96I) has reported a variety of abnormalities in the plasma proteins of schizophrenic patients as shown by electrophoretic methods, particularly in the globulin fraction already incriminated, it will be recalled, by the work of Frohman and his co-workers (1960, a, b, c) on intermediate carbohydrate metabolism and Bergen and others (1960) on the toxic factor in plasma.

\section{Summary and Discussion}

One main finding of recent research has been the demonstration of the close chemical relationship between important constituents of the brain and hallucinogenic agents. Catecholamines and tryptamine derivatives such as $5-\mathrm{HT}$ are found in those parts of the brain concerned with the highest control of the autonomic nervous system. Simple chemical modification of these and similar compounds according to systematic principles (Omethylation and N-methylation) leads to the production of potent psychotomimetic agents such as mescaline, DMT, psilocybin and (to some extent) bufotenin. Furthermore, most psychotomimetic agents seem to act as central stimulants of the sympathetic nervous system and as peripheral sensitizing agents to the action of adrenaline. Brain HT may further be involved in psychosis as is suggested by the observed correlation between the ability of LSD and its analogues to cause psychosis and their power to raise levels and rates of turnover of $5-\mathrm{HT}$ in the brain.

Confirmation of the relation between excess methylation and psychosis has been obtained by observing that two methyl donors-methionine and betaine-both aggravate the symptoms of schizophrenic subjects. It would be interesting to repeat these experiments in normal people. Further supportive evidence has been obtained in the (as yet unconfirmed) reports that bufotenin and dimethoxyphenylethylamine have been isolated by chromatographic methods from schizophrenic urine. Thus a number of different leads all cross at the same point and enable us to make the working hypothesis that the most promising field to study in schizophrenia would be that of methylation processes as applied to catecholamine and tryptamine derivatives. The way is now open for a considerable number of critical experiments to test this hypothesis and progress in this field should be rapid.

Also of interest are the changes in intermediate carbohydrate metabolism, tryptophane metabolism and neuraminic acid levels that have been reported. Further developments in these fields also can be expected as well as attempts to correlate one field with another. One could ask such questions as ' would changes in methylation processes be expected to affect intermediate carbohydrate metabolism and if so how'; and vice versa. Vigorous attempts are at present being made to isolate and identify the 'toxic factor' in schizophrenic plasma. Its location in the globulin fraction offers the possibility of correlation with the changes recently discovered in the immunological properties of schizophrenic plasma.

But clearly priority in research at present should go to further testing that particular 
hypothesis that has already withstood the most critical tests. This is the hypothesis of excess methylation. Priority should also go to basic studies of the neurochemical and neuropharmacological mode of action of the known chemical compounds that induce in man a state resembling psychosis. This requires multidisciplinary research on a large scale. If only we can discover some of the biochemical mechanisms that underlie psychosis then we can test the performance of these mechanisms in schizophrenia. Furthermore, if we know how mescaline, LSD, DMT, etc., act, we could design anti-metabolites using rational principles to block their action and we could then give these drugs empirical trial in schizophrenia.
It is possible, for example, that in order to be a psychotomimetic agent a drug must both stimulate the central sympathetic and sensitize tissues to the action of the catecholamines. Any drug doing only one or the other is ineffective. Drugs which are effective in treating schizophrenia-such as chlorpromazine-certainly depress central sympathetic function and have anti-adrenaline properties. Amphetamine, on the other hand, is a powerful stimulant of the central sympathetic system and can induce in certain people a typical paranoid schizophrenic illness. Basic research of this kind leads eventually to therapeutic trials and the progress from cause to therapy is, after all, the aim of medical research.

\section{REFERENCES}

Armstrong, M. D., McMillan, A., and Shaw, K. N. F. (1957): 3-Methoxy-4-hydroxy-D-mandelic Acid, a Urinary Metabolite of Norepinephrine, Biochim. biophys. Acta., 25, 422.

Arnold, O. H., and Hofmann, G. (1962): Der intermediäre Phosphatstoffwechsel des Erythrozyten bei Normalpersonen, Schizophrenen und deren Familienangehörigen unter Bernsteinsäurebelastung, Wien. Z. Nervenheilk., I9, 15.

AshCROFT, G. (1962): Personal communication.

AXELROD, J. (196r): Enzymatic formation of Psychotomimetic Metabolites from Normally Occurring Compounds, Science, 134, 343. Benassi, C. A., Benassi, P., Allegri, G., and Ballarin, P. (196I): Tryptophan Metabolism in Schizophrenic Patients Y. Neurochem., 7, 264 .

Bergen, J. R., Pennell, R. B., Freeman, H., Hoagland, H., and Smythies, J. R. (1960): Rat Behaviour Changes iff Response to a Blood Factor from Normal and Psychotic Persons, Arch. Neurol. Psychiat. (Chicago), $2,146$.

-, Koella, W. P., Czicmann, J., Hoagland, H. (196r): Evoked Optic Potential Changes Induced by Plasma Globulins from Schizophrenics, Fed. Proc., 20, 305.

Bishop, M. B. (1960): Effect of Schizophrenic Plasma Upon Original Learning in the Rat, Dis. nerv Syst., 2 r, r33.

Bogoch, S., Dussik, K. T., and Lever, P. G. (1959): Clinical Status and Cerebrospinal Fluid 'Total Neuraminic Acid', A.M.A. Arch. gen. Psychiat., 1, 441.

Brodie, B. B. (1958): Storage and Release of 5-Hydroxytryptamine, in '5-Hydroxytryptamine' (edited by G. P. Lewis), p. 64. London: Pergamon Press.

Brown, F. C., White, J. B., and KenNedY, J. K. (1960): Urinary Excretion of Tryptophan Metabolites by Schizophrenic Individuals, Amer. F. Psychiat., I17, 63.

Brune, G. G., and Himwich, H. E. (1962): Indole Metabolites in Schizophrenic Patients, A.M.A. Arch. gen. Psychiat., 6,82 .

(1962): Biogenic Amines and Behaviour in Schizophrenic Patients, in 'Recent Advances in Biological Psychiatry', p. 5. In the press.

Christoni, G., and Zappoli, R. (1960): Neuraminic Acids in the Cerebrospinal Fluid of Schizophrenic and Oligophrenic Patients, Amer. F. Psychiat., II7, 246.

Cohen, G., Holland, B., and Goldenberg, M. (r959): Disappearance Rates of Infused Epinephrine and Norepinephrine from Plasma, A.M.A. Arch. gen. Psychiat., $\mathrm{x}, 228$.

Costa, E. (1960): The Role of Serotonin in Neurobiology, Int. Rev. Neurobiol., 2, 137.

Fessel, W. J. (196r): Disturbed Serum Proteins in Chronic Psychosis. Serological, Medical and Psychiatric Correlations, A.M.A. Arch. gen. Psychiat., 4, 154.

Fischer, E., Lagravere, T. A. F., Vazquez, A. J., and Di Stefano, A. O. (196r): A Bufotenin-like Substance in the Urine of Schizophrenics, $\mathcal{Y}$. nerv. ment. Dis., 133,441 .

Freedman, D. X. (196r): Effects of LSD-25 on Brain Serotonin, Y. Pharmacol., 134, 160.

Friedhoff, A. J., and VAN WinkLe, E. (1962): Isolation and Characterization of a Compound from the Urine of Schizophrenics, Nature, r94, 897.

Frohman, C. E., Latham, L. K., Beckett, P. G. S., and Gottlieb, J. S. (r96oa): Evidence of a Plasma Factor in Schizophrenia, A.M.A. Arch. gen. Psychiat., 2, 255.

-, Czajkowski, N. P., Luby, E. D., Gotrlieb, J. S., and Senf, R. (1960b): Further Evidence of a Plasma Factor in Schizophrenia, Ibid., 2, 263.

-, Luby, E. D., Tourney, G., Becketr, P. G. S., and Gottlieb, J. S. (I96oc): Steps Towards the Isolation of a Serum Factor in Schizophrenia, Amer. F. Psychiat., Ix7, 401.

Gaddum, J. H. (1954): Drugs Antagonistic to 5-Hydroxytryptamine, in 'Ciba Foundation Symposium on Hypertension' (edited by G. E. W. Wolstenholme and M. P. Cameron). Boston: Little, Brown.

Geiger, R. S. (1960): Effects of LSD-25, Serotonin and Sera from Schizophrenic Patients on Adult Mammalian Brain Cultures, $\mathcal{Y}$. Neuropsychiat., 1,185 .

German, G.'A. (1961): Serum from Schizophrenics and Cortical Potentials in the Rat, F. Physiol., 160, roP. 
Haddad, R. K., and Rabe, A. (1961): 'An Anaphylactic Test for Abnormal Antigen(s) in Schizophrenic's Serum ', Abstracts IIIrd World Congress of Psychiatry, Montreal, p. 608.

Harley-Mason, J., Laird, A., and Smythies, J. R. (1958): The Metabolism of Mescalin in the Human, Confin. neurol. (Basel), 18, 152.

La Brosse, E. H., ManN, J. D., and Kety, S. S. (196r): The Physiological and Psychological Effects of Intravenously Administered Epinephrine and its Metabolism in Normal and Schizophrenic Men-III. Metabolism of $7-\mathrm{H}^{3}$ epinephrine as Determined in Studies of Blood and Urine, Y. Psychiat. Res., I, 68.

LENZ, H. (1962): Papierchromatographische Untersuchungen des Harnes bei Schizophrenen und Geistesgesunden vor und nach Dopa-Verabreichung, Psychopharmacologia, 3, 146.

LuCY, J. D. (1954): Histamine Tolerance in Schizophrenia, Arch. Neurol. Psychiat. (Chicago), 7I, 629.

Ludueña, F. P. (1935): Sombre la farmacologia de la tricocereina, alcaloide del Trichocereus Terscheki (Parm.) Britton et Rose, Rev. Soc. argent. Biol., 11, 604.

McIsaAc, W. M. (196I): A Biochemical Concept of Mental Disease, Postgrad. Med., 30, i 1 I.

Pollin, W., Cardon, P. V. Jr., and Kety, S. S. (196r): Effects of Amino Acid Feedings in Schizophrenic Patients Treated with Iproniazid, Science, 133, 104.

— and GoldiN, S. (196r): The Physiological and Psychological Effects of Intravenously Administered Epinephrine and its Metabolism in Normal and Schizophrenic Men-II. Psychiatric Observations, F. Psychiat. Res., I, 50.

Price, J. M., Brown, R. R., and Peters, H. A. (1959): Tryptophan Metabolism in Porphyria, Schizophrenia and a Variety of Neurologic and Psychiatric Diseases, Neurology, 9, 456.

Resnick, O., Wolfe, J. M., Freeman, H., and Elmadjian, F. (I958): Iproniazid Treatment and Metabolism of Labelled Epinephrine in Schizophrenics, Science, 127, I 116.

RodNight, R. (x961): Body Fluid Indoles in Mental Illness, Int. Rev. Neurobiol., 3, 251.

SACKs, W. (1959): Cerebral Metabolism of Isotopic Glucose in Chronic Mental Disease, f. applied Physiol., I4, 849.

Sankar, D. V. S., Gold, E., Phipps, E., and Sankar, D. B. (I96r) : ' Biochemical Studies on Schizophrenic Children', Abstracts IIIrd World Congress of Psychiatry, Montreal, p. 202.

SmythiEs, J. R. (i963): ' Schizophrenia. Biochemistry, Metabolism and Treatment'. Springfield, Illinois: Chartes C Thomas.

- Koella, W. P., and Levy, C. (1960): The Effect of Mescalin on the Optic Evoked Potentials in the Unanesthetized Rabbit, F. Pharmacol., 129, 462.

WADA, J., and Gibson, W. C. (1959): Behavioral and EEG Changes Induced by Injection of Schizophrenic Urine: Extract, Arch. Neurol. Psychiat. (Chicago), 81, 747.

Winter, C. A., and Flataker, L. (1958): Effect of Blood Plasma from Psychotic Patients upon Performance of Trained Rats, Ibid., 80, 44I.

Woolley, D. W., SHAW, E. (r954): A Biochemical and Pharmacological Suggestion About Certain Mental Disorders, Proc. nat. Acad. Sci. (Wash.), 40, 228. 